\title{
Comparing Pediatric Deaths With and Without Hospice Support
}

\author{
David S. Dickens*
}

Background. Although pediatric hospice care is commonly accepted as a beneficial intervention, the incremental advantage over end-of-life care delivered without engaging hospice remains unknown. The primary objective of this study was to describe differences in pediatric end-of-life care when delivered with and without hospice support, as perceived by the medical provider. Procedure. A retrospective survey of medical providers was conducted in 2005 over a 2-month period at a single institution, the Helen DeVos Children's Hospital (HDVCH). Medical providers with self-determined experience in pediatric end-of-life care were asked to separately provide positive and negative comments about their experience with hospice. Additionally they were asked to describe differences between children under their supervision who died with and without hospice care. Medical provider comments and comparisons of experiences in caring for children dying with and without hospice involvement are described. Results. Out of 157 responders, 43 reported positive comments about the hospice intervention. Non-medical support and location of death were the most frequently cited benefits. Nineteen responders provided negative comments about hospice; all involving feelings of lost hope, intrusion, or distrust. When asked to directly compare deaths with and without hospice support, 44 of $51(86 \%)$ responders favored hospice. The most cited reason for preferring hospice involvement was better provision of non-medical services. Conclusions. The majority of pediatric providers in this survey observed an advantage to utilizing hospice care for dying children as compared to providing end-of-life care without hospice involvement. Pediatr Blood Cancer 2010;54:746-750. (๑) 2010 Wiley-Liss, Inc.

Key words: childhood death; comparative effectiveness; pediatric hospice; pediatric palliative care

\section{INTRODUCTION}

Most dying children experience symptoms that negatively impact quality of life [1-4]. Best practices that treat pain and alleviate symptoms during end-of-life care are a matter of ongoing research. Although hospice and palliative care services have shown to reduce symptoms, improve quality of life, and impact the perception of quality end-of-life care in adults [5-7], few data are available showing similar benefit in children [8-10]. Despite the limited data, the Institute of Medicine, the American Academy of Pediatrics and many experts in the field strongly advocate for the integration of palliative and hospice care for children [11-13]. If it becomes accepted that palliative and hospice care is desirable, the question then becomes: Who is going to deliver such a service? When it comes to providing care for terminally ill children, pediatricians report feeling inadequately educated, variably confident, and unlikely to access training to improve their ability [1416]. Even within a subspecialty exposed to a relatively high patient mortality rate such as oncology, physicians have reported inconsistent preparation for, and comfort with, providing comprehensive end-of-life care [17-19]. Between the promoted benefits of hospice care and the discomfort medical providers express in delivering end-of-life care, one could expect near universal enrollment of dying children onto hospice programs. However, this does not appear to be the case.

In part, low hospice enrollment may be due to limited availability. Recent research shows that roughly $60 \%$ of institutions participating in the Children's Oncology Group have hospice programs available to them [12]. The challenge of limited hospice availability is compounded by poor utilization. In the above-cited survey, the median number of hospice enrollments was less than half of the number of patient deaths. Such underutilization of hospice services has also been observed in non-oncology patients [20] as well as other pediatric oncology practices worldwide [21,22]. In an attempt to explain underutilization, Davies et al. explored pediatric provider perceptions on barriers to hospice enrollment. The most frequently encountered responses included uncertain prognosis, family preferences, language barriers, and time constraints of the medical provider [23].

(C) 2010 Wiley-Liss, Inc.

DOI 10.1002/pbc.22413

Published online 8 January 2010 in Wiley InterScience (www.interscience.wiley.com)
Uncertain prognosis and family preference are significant obstacles inherent to the practice of pediatric oncology. Beyond these issues, the other identified barriers to hospice enrollment are less formidable. Language differences and time constraints are not matters unique to hospice enrollment. These variables frequently impact decision-making and are surmountable when there is a commitment to the intervention being recommended. It therefore becomes unclear whether there is a common belief among medical providers that hospice offers significant benefit to the incurable. To this point, there is a need to study outcome, however, it may be defined, between patients who die with and without hospice support. All previous studies showing benefit to hospice only compare outcomes in the individual patient before and after enrollment. This survey was conducted in part to collect observations from medical providers experienced in pediatric end-of-life care, allowing them to compare their experiences caring for dying children with and without hospice support.

Division of Pediatric Hematology/Oncology and Bone Marrow Transplantation, Department of Pediatrics \& Human Development, Helen DeVos Children's Hospital, Michigan State University College of Human Medicine, Hospice of Michigan, Michigan

Conflict of interest: The author has served as the medical director for the hospice team primarily utilized by the community at the time the study was conducted through the present. Although no direct compensation has ever been received, Hospice of Michigan gives the author's primary employer $\$ 10,000 /$ year for salary support. Author also serves on the Foundation Board of Directors for Hospice of Michigan.

Assistant Professor; Medical Director of Pediatric Programs.

*Correspondence to: David S. Dickens, Division of Pediatric Hematology/Oncology and Bone Marrow Transplantation, Helen DeVos Children's Hospital, 100 Michigan St., Grand Rapids, MI 49503. E-mail: david.dickens@devoschildrens.org

Received 25 September 2009; Accepted 1 December 2009 


\section{METHODS}

\section{Setting}

An electronic, 34-question survey was opened for a 2-month period in 2005 at the Helen DeVos Children's Hospital (HDVCH); a 190-bed, tertiary-care teaching hospital located in western Michigan. The survey was submitted to and approved by the local institutional review board. At the time, HDVCH served as a referral center for 37 Michigan counties with $\sim 100$ physicians in 40 pediatric specialties on staff. The primary hospice provider utilized by the study population was Hospice of Michigan (HOM)'s pediatric team which includes a physician, nurses, social workers, and a spiritual care provider dedicated exclusively to pediatrics. Although the majority of patients had their care delivered in their own homes, inpatient care was made available at $\mathrm{HDVCH}$ if symptoms were uncontrollable. During the calendar year of the survey, HOM provided hospice care to 25 children. Six of these patients had a primary oncologic diagnosis, nine patients had a primary neurologic diagnosis, and six had a genetic or metabolic diagnosis. Other primary diagnoses included pulmonary and renal. HOM's pediatric program has an early care service which functions similar to a traditional palliative care program. Patients with life-limiting illnesses and no foreseeable terminal events can enroll while pursuing curative therapies. They are given access to all aspects of hospice care: psychosocial, emotional, and spiritual support, with the exception that no medical intervention is offered.

\section{Survey Development and Implementation}

The survey contained demographic, knowledge-based opinion, and practice experience items. Questions were developed from a combination of existing needs assessment surveys, quality research studies, and clinical experience [24,25]. The survey was then piloted by five individuals with end-of-life care experience including the author. Minor adjustments were made to the survey prior to the institutional release. Participants were alerted about the survey via a hospital-wide e-mail and hand delivered paper notifications. Two e-mail reminders were sent during the collection phases.

\section{Sample and Data Collection}

Responses were requested from staff with self-determined experience in caring for children with terminal conditions. The survey tool did not require all questions to be answered. The focus of this review was to collect comments, positive or negative, about the responders' experiences with hospice. Additionally, responders were asked to compare their experiences with dying children who did and did not receive hospice care. Questions included: (1) Have you received positive feedback from a family of a child who has been enrolled on hospice care? If yes, please explain. (2) Have you received negative feedback from a family of a child who has been enrolled on hospice care? If yes, please explain. (3) Have you had patients die both on hospice care, and not on hospice care? If yes, have you observed any differences? Responders were allowed to provide comments in an open, free-text format. Frequently, multiple comments were received per question and therefore the total number of comments exceeds the total number of responders. Comments were studied for content, collated into several general categories, and reported accordingly.

\section{Statistical Analysis}

Following the primary objective of describing the differences between children who died with and without hospice, the data analysis was performed utilizing descriptive statistics.

\section{RESULTS}

A total of 157 providers completed the survey. The discipline with the highest number of responders was nursing $(n=71 ; 45 \%)$, followed by physicians $(n=45 ; 29 \%)$. The response rate was estimated to be at least $10 \%$ for nurses and $45 \%$ for physicians. As only individuals with self-determined pediatric end-of-life care experience were asked to respond, the true response rate is unobtainable. Other disciplines represented in this survey included nurse practitioners and physician assistants $(n=15 ; 9 \%)$, social workers $(n=11 ; 7 \%)$, and one chaplain. Of those who identified their specialty area, most were within the hematology/oncology and bone marrow transplantation $(\mathrm{H} / \mathrm{O}+\mathrm{BMT})$ division $(\mathrm{n}=45 ; 29 \%)$, followed by general pediatrics $(n=37 ; 24 \%)$, neonatal intensive care $(n=27 ; 17 \%)$ and pediatric intensive care $(n=16 ; 10 \%)$. The majority of general pediatric responders worked in a hospital-based practice $(n=26 ; 70 \%)$ versus an ambulatory setting $(n=11 ; 30 \%)$. Other specialties represented at $<5 \%$ of total responders included nephrology, pulmonology, and neurology. Further details on the responders' demographics have been previously published [26].

Forty-three responders provided 70 positive comments (Table I). Most responders were physicians $(n=21 ; 49 \%)$ followed by nurses $(\mathrm{n}=10 ; 23 \%)$ and physician assistants/nurse practitioners $(\mathrm{n}=7 ; 16 \%)$. Hospital-based practices represented the majority of responders who provided positive comments $(n=35 ; 81 \%)$. The predominant specialty was $\mathrm{H} / \mathrm{O}+\mathrm{BMT}(\mathrm{n}=16 ; 37 \%)$. Other specialties were almost equally represented at much lower percentages. Most positive comments involved issues of nonmedical support. Some comments were non-specific and reported

\section{TABLE I. Comments Received on Hospice Care}

\begin{tabular}{lccc}
\hline & Positive $(27 \%)$ & & Negative (12\%) \\
\cline { 2 - 2 } Category & 43 responders & & 19 responders \\
\hline Non-medical support & 22 & \\
Location of death & 10 & \\
Skill of provider & 7 & \\
Non-specific & 7 & \\
Preparation for death & 6 & \\
Dignity/peace/quality & 6 & \\
Symptom control & 5 & \\
Coping & 3 & \\
Decision-making & 3 & \\
Care coordination & 1 & \\
Misperception of services & & \\
Distrust & & \\
Hope & & \\
Intrusion & 70 & \\
Total number of comments & & \\
\hline
\end{tabular}

${ }^{\mathrm{a}}$ Non-specific was used as a category when the responder stated to have heard or observed positive things, but did not elaborate on what exactly was positive about the experience; ${ }^{\mathrm{b}}$ Number of comments exceeds number of responders as multiple observations were allowed. 
that the family felt "better supported" or that the child's death was "peaceful and dignified." The ability to have the child cared for at home was the most commonly received specific positive comment made about the hospice intervention. Other specific positive comments included satisfaction with symptom control, quality of life, skill of the hospice staff, coordination of care, assistance with decision-making, and family preparedness.

Of the 19 responders who reported negative comments, there were 11 physicians, 3 social workers, 3 physician extenders, and 2 nurses. Most were from the H/O + BMT division $(\mathrm{n}=12 ; 63 \%)$, including five physicians. Seven negative comments involved a misperception of services (Table I). These included expectations for a respite home and/or continuous nursing presence in the home, confusion over after-hour coverage, and heightened expectations for symptom control. Seven responders expressed issues of distrust between families and hospice staff. Based on several responses, trust was a particular issue for families who experienced a limited time on hospice prior to a child's death, a perceived limited experience among the hospice staff, and/or a loss of contact with primary caregivers. Five responders felt that hospice enrollment diminished hope. Three comments indicated that the hospice intervention felt too intrusive for the family.

Central to this study's objective, 51 responders (32\%) offered direct comparisons between children who died with and without hospice support (Table II). Nurses $(\mathrm{n}=20 ; 40 \%)$ and physicians $(\mathrm{n}=19 ; 37 \%)$ represented the majority of responders to this question, most of whom practiced in the $\mathrm{H} / \mathrm{O}+\mathrm{BMT}$ division $(n=19 ; 37 \%)$. The majority of responders $(n=44 ; 86 \%)$ reported an advantage to having hospice involved during end-of-life care. The specific comments indicated that providers observed better psychosocial and emotional support as well as improved symptom control when hospice was involved. Additionally, providers reported that dying children enrolled on hospice services had better preparation for death, preferable location of death, superior care coordination and died more peacefully with less chaos and more dignity.

Five responders (10\%) reported no perceived difference and, in all cases, were equally positive about the care delivered. There were two responders who observed hospice to be less preferable. Both of these responders were experienced, hospital-based subspecialists who commented that under some circumstances, the hospice service felt like an intrusion of new care providers into a delicate and private situation. Representative positive, negative, and comparative comments are presented in Table III.

\section{DISCUSSION}

Reported studies documenting improvements in pain management, symptom control, and quality of life with hospice support have been limited to comparisons for individual patients before and after hospice enrollment [5-7]. Clinicians practicing in comprehensive care settings may argue that patients discontinuing curative therapy may achieve similar improvements in symptom control and quality of life without formal hospice support. In contrast to previous reports, this study offers a direct comparison between children who die with and without hospice as perceived by medical providers. By doing so, incremental benefits of the hospice intervention can begin to be discerned. This study's findings indicate a strong preference by medical providers for the hospice intervention. Better psychosocial services, anticipatory grief support, and superior care coordination were major benefits observed in patients who received hospice care. Better symptom control and preferred location of death were also frequently observed advantages.

These results stimulate a more defining question than "Is hospice better," and that is "Why is hospice better." The reason most frequently cited by responders was the ability to remain in a home setting with hospice involvement. While location itself was identified in this study as an independent factor favoring the hospice intervention, there were other stated benefits of hospice likely to be influenced by the location of death, including less chaos, fewer interventions and more dignity. HDVCH has well developed resources for care coordination, symptom control, and psychosocial support. It is unclear why these variables were perceived as delivered comparatively better when patients were enrolled on hospice care. It is possible that having a separate team dedicated to end-of-life care creates better focus. It is also possible that the traditional medical team is inadequately trained, equipped or prepared to provide such multidisciplinary care for the dying child and their families. The difference may simply be that the patients and families who chose hospice were better able to accept symptom control, grief, and psychosocial support as goals and work towards them with resolve.

The negative comments received were extremely helpful from a service delivery and needs assessment perspective. Loss of hope is a complex, frequently encountered barrier that in the author's experience is most effectively handled by the referring care providers, not the hospice team. Education of referral sources and the family at the time of referral could potentially help set more

TABLE II. Comments Comparing Hospice to Non-Hospice Deaths

\begin{tabular}{|c|c|c|c|}
\hline & Favoring hospice & Favoring no. hospice & No difference \\
\hline Category & 44 responders $(86 \%)$ & 2 responders $(4 \%)$ & 5 responders $(10 \%)$ \\
\hline Non-medical support & 25 & & \\
\hline Symptom control/comfort & 20 & & \\
\hline Preparation for death & 9 & & \\
\hline Location of death & 8 & & \\
\hline Care coordination & 8 & & \\
\hline Peace/less chaos & 6 & & \\
\hline Dignity & 2 & & \\
\hline Intrusion & & 2 & \\
\hline Total number of comments ${ }^{\mathrm{a}}$ & 78 & 2 & 5 \\
\hline
\end{tabular}

${ }^{\mathrm{a}}$ Number of comments exceeds number of responders as multiple observations were allowed. 
TABLE III. Specific Comments Received on Hospice Care

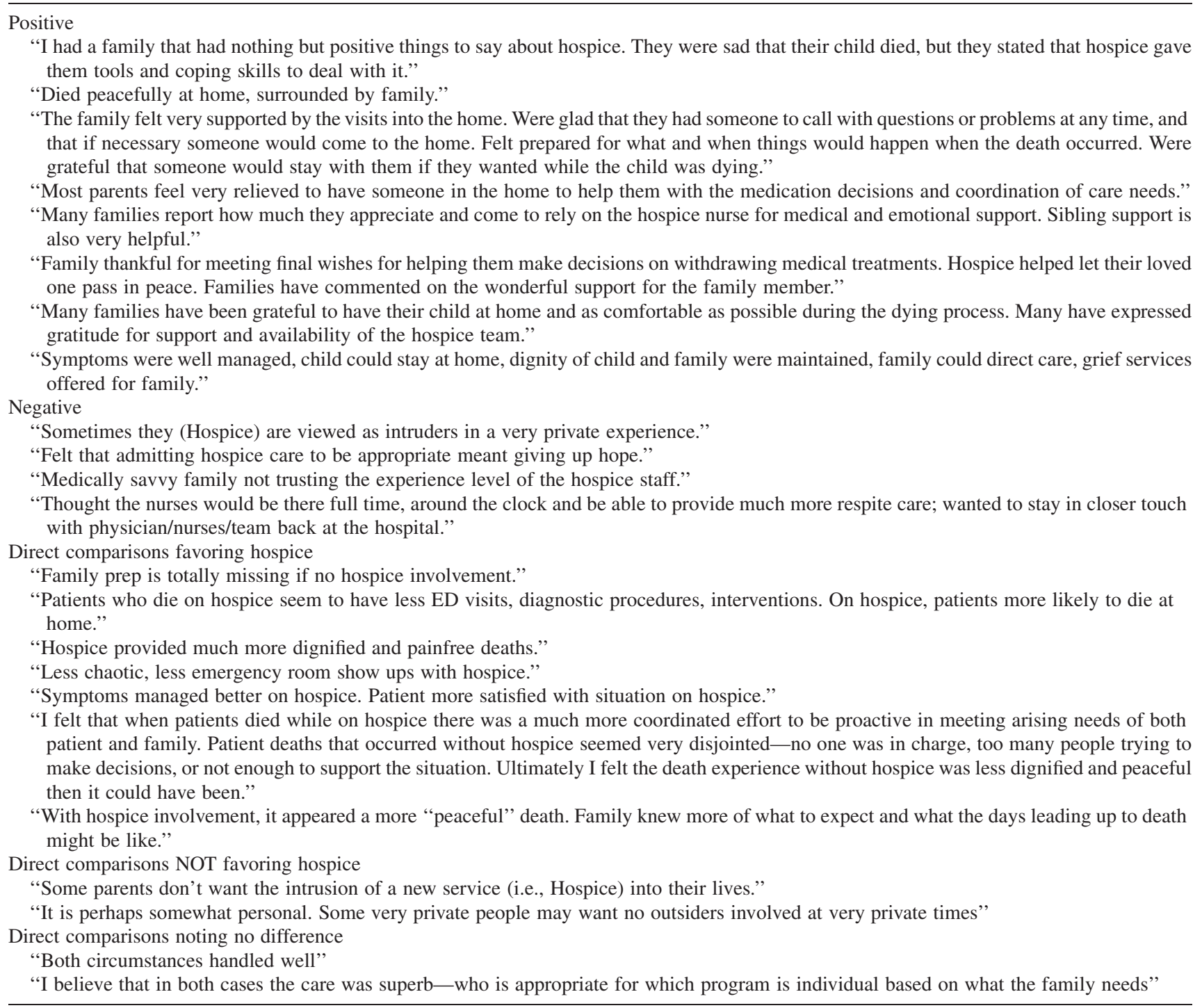

realistic expectations. The identification of intrusion and distrust as being hospice dissatisfiers could be used as further evidence for the importance of palliative care programs. Palliative care allows for patients and their families to enter the hospice system earlier, as the traditional barriers to hospice enrollment (discontinuation of curative therapy and defining a life expectancy) are removed. This earlier intervention could then allow more time for trust building and potentially lead to fewer feelings of intrusion. However, the limited presence and usage of dedicated palliative care programs prevent this from becoming more than a partial solution. With regard to the responders who reported no difference on direct comparison, all were hospital-based providers. The roles and responsibilities of these five responders (two hospitalists, two inpatient oncology nurses, and one inpatient nurse practitioner) do not include outpatient follow-up, perhaps making it difficult for these individuals to detect non-medical impact.

The results of this study need to be interpreted within the context of several limitations. Low response rates and the single institution design increase the possibility that this study's findings do not represent the opinions of pediatric medical providers in general. The characteristics of non-responders were not collected, introducing the possibility of responder bias. The responder population was overrepresented by the H/O + BMT discipline. Staff from this specialty represented $29 \%$ of responders, but only $\sim 6 \%$ of total employed staff, which may bias the experience level and the capacity to observe and manage patients on a hospice service. Conversely, while $36 \%$ of the hospice census at the time of the survey was comprised of patients with primary neurologic diagnoses, only $4 \%$ of responders to the survey identified themselves as working in neurology. Recall and reporter biases inherent to the retrospective design and survey format could also potentially misrepresent clinical realities. Lastly, the community that was surveyed primarily utilizes a single hospice provider which offers a team dedicated exclusively to pediatrics, a level of service which may be unavailable elsewhere.

In spite of these limitations, this study has value in that it provides a direct comparison of children who die with and without hospice support. Evidence is provided that hospice as an 
intervention offers benefit over the alternative, which is to not have hospice involved during end-of-life care. A larger, multi-institutional, prospective study would be necessary to eliminate as many confounding variables as possible, maximizing the chance that the only major difference between the study populations is hospice enrollment itself. Patients would need to be selected at the time that the discontinuation of curative therapies is recommended by their primary medical team. Comparisons collected on patients who choose and do not choose hospice could include medical, psychosocial, and emotional outcomes as well as provider/patient/ family satisfaction with variables assessed in this study.

Even with emerging evidence and advocacy that promote pediatric hospice and palliative care as being beneficial, these services remain underutilized. Attempts at overcoming barriers could remain ineffective unless evidence exists that hospice intervention can offer care, comfort, and positive outcomes that cannot be otherwise obtained.

\section{ACKNOWLEDGMENT}

The author would like to thank Hospice of Michigan for the support of their dedicated and accomplished pediatric team including; Mary Spicketts RN, Dawn Vanderkolk LMSW, Sara Blue RN, Ruth-Anne Belch RN, Marlo Schepers RN, Cheryl Lamport DMin, and Sheila Schindler LMSW. Appreciation is extended to those who participated in the survey and the Spectrum Health Research Department for their assistance in conducting the study, in particular Teri Crumb, RN, BSN, CCRC. Special thanks to James Fahner, MD for his mentorship and critical review.

\section{REFERENCES}

1. Hechler T, Blankenburg M, Friedrichsdorf SJ, et al. Parents' perspective on symptoms, quality of life, characteristics of death and end-of-life decisions for children dying from cancer. Klin Padiatr 2008;220:166-174.

2. Wolfe J, Grier HE, Klar N, et al. Symptoms and suffering at the end of life in children with cancer. N Engl J Med 2000;342: $326-333$.

3. Theunissen JM, Hoogerbrugge PM, van Achterberg T, et al. Symptoms in the palliative phase of children with cancer. Pediatr Blood Cancer 2007;49:160-165.

4. Pritchard M, Burghen E, Srivastava DK, et al. Cancer-related symptoms most concerning to parents during the last week and last day of their child's life. Pediatrics 2008;121:e1301-e1309.

5. Stromgren AS, Sjogren P, Goldschmidt D, et al. A longitudinal study of palliative care: Patient-evaluated outcome and impact of attrition. Cancer 2005;103:1747-1755.

6. Strasser F, Sweeney C, Willey J, et al. Impact of a half-day multidisciplinary symptom control and palliative care outpatient clinic in a comprehensive cancer center on recommendations, symptom intensity, and patient satisfaction: A retrospective descriptive study. J Pain Symptom Manage 2004;27:481-491.

7. Follwell M, Burman D, Le LW, et al. Phase II study of an outpatient palliative care intervention in patients with metastatic cancer. J Clin Oncol 2009;27:206-213.
8. Wolfe J, Hammel JF, Edwards KE, et al. Easing of suffering in children with cancer at the end of life: Is care changing? J Clin Oncol 2008;26:1717-1723.

9. Golan H, Bielorai B, Grebler D, et al. Integration of a palliative and terminal care center into a comprehensive pediatric oncology department. Pediatr Blood Cancer 2008;50:949-955.

10. Knapp CA, Madden VL, Curtis CM, et al. Partners in care: Together for kids: Florida's model of pediatric palliative care. J Palliat Med 2008;11:1212-1220.

11. American Academy of Pediatrics. Palliative care for children. Committee on Bioethics and Committee on Hospital Care. Vol. 106. ELK Grove Village, Illinois: American Academy of Pediatrics; 2000. pp. 351-357.

12. Johnston DL, Nagel K, Friedman DL, et al. Availability and use of palliative care and end-of-life services for pediatric oncology patients. J Clin Oncol 2008;26:4646-4650.

13. Institute of Medicine. Special Issues in Pediatric Oncology. Gelb K, editor. Improving Palliative Care for Cancer. Washington DC: National Academy Press; 2001. pp. 161-198.

14. McCabe ME, Hunt EA, Serwint JR. Pediatric residents' clinical and educational experiences with end-of-life care. Pediatrics 2008; 121:e731-e737.

15. Kolarik RC, Walker G, Arnold RM. Pediatric resident education in palliative care: A needs assessment. Pediatrics 2006;117:1949_1954.

16. Sheetz MJ, Bowman MA. Pediatric palliative care: An assessment of physicians' confidence in skills, desire for training, and willingness to refer for end-of-life care. Am J Hosp Palliat Care 2008;25:100-105.

17. Fowler K, Poehling K, Billheimer D, et al. Hospice referral practices for children with cancer: A survey of pediatric oncologists. J Clin Oncol 2006;24:1099-1104.

18. Hilden JM, Emanuel EJ, Fairclough DL, et al. Attitudes and practices among pediatric oncologists regarding end-of-life care: Results of the 1998 American Society of Clinical Oncology survey. J Clin Oncol 2001;19:205-212.

19. Roth M, Wang D, Kim M, et al. An assessment of the current state of palliative care education in pediatric hematology/oncology fellowship training. Pediatr Blood Cancer 2009;53:647-651.

20. Knapp CA, Thompson LA, Vogel WB, et al. Developing a pediatric palliative care program: Addressing the lack of baseline expenditure information. Am J Hosp Palliat Care 2009;26:4046.

21. Widger K, Davies D, Drouin DJ, et al. Pediatric patients receiving palliative care in Canada: Results of a multicenter review. Arch Pediatr Adolesc Med 2007;161:597-602.

22. Friedrichsdorf SJ, Menke A, Brun S, et al. Status quo of palliative care in pediatric oncology-a nationwide survey in Germany. J Pain Symptom Manage 2005;29:156-164.

23. Davies B, Sehring SA, Partridge JC, et al. Barriers to palliative care for children: Perceptions of pediatric health care providers. Pediatrics 2008;121:282-288.

24. Andresen EM, Seecharan GA, Toce SS. Provider perceptions of child deaths. Arch Pediatr Adolesc Med 2004;158:430-435.

25. Solomon MZ, Sellers DE, Heller KS, et al. New and lingering controversies in pediatric end-of-life care. Pediatrics 2005;116: 872-883.

26. Dickens DS. Building competence in pediatric end-of-life care. J Palliat Med 2009;12:617-622. 